\title{
Distribution of dyssynchrony in subjects with no known cardiac disease and comparison of velocity vector imaging to color-coded tissue Doppler imaging.
}

\author{
Anoop C Parameswaran \\ St. John's Health System \\ Bhaskar Purushottam \\ Einstein Medical Center \\ Aman Amanullah \\ St. John's Health System \\ Vincent M. Figueredo, M.D.

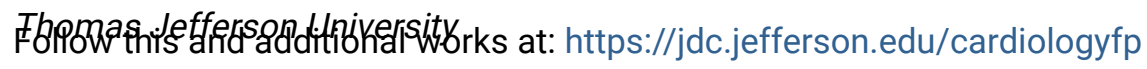 \\ Part of the Cardiology Commons \\ Let us know how access to this document benefits you
}

\section{Recommended Citation}

Parameswaran, Anoop C; Purushottam, Bhaskar; Amanullah, Aman; and Figueredo, M.D., Vincent M., "Distribution of dyssynchrony in subjects with no known cardiac disease and comparison of velocity vector imaging to color-coded tissue Doppler imaging." (2013). Division of Cardiology Faculty Papers. Paper 56.

https://jdc.jefferson.edu/cardiologyfp/56

This Article is brought to you for free and open access by the Jefferson Digital Commons. The Jefferson Digital Commons is a service of Thomas Jefferson University's Center for Teaching and Learning (CTL). The Commons is a showcase for Jefferson books and journals, peer-reviewed scholarly publications, unique historical collections from the University archives, and teaching tools. The Jefferson Digital Commons allows researchers and interested readers anywhere in the world to learn about and keep up to date with Jefferson scholarship. This article has been accepted for inclusion in Division of Cardiology Faculty Papers by an authorized administrator of the Jefferson Digital Commons. For more information, please contact: JeffersonDigitalCommons@jefferson.edu. 
Distribution of dyssynchrony in subjects with no known cardiac disease and comparison of velocity vector imaging to color coded tissue Doppler imaging.

Anoop C. Parameswaran MD, MPH ${ }^{\mathrm{a}}$, Bhaskar Purushottam MD ${ }^{\mathrm{b}}$, Aman Amanullah MD, $\mathrm{PhD}^{\mathrm{b}}$, Vincent M. Figueredo $\mathrm{MD}^{\mathrm{b}}$

(a) St. John's Health System, Springfield, MO

(b) Einstein Institute for Heart and Vascular Health, Einstein Medical Center and Jefferson Medical College, Philadelphia, PA

Running head: Dyssynchrony in subjects with no known cardiac disease

Conflict of Interest: The authors have no conflicts of interest to report.

Key Words: Dyssynchrony, Velocity Vector Imaging, color coded tissue Doppler imaging

Corresponding Author: Vincent M. Figueredo, MD, FASE

Professor of Medicine, Jefferson Medical College

Director, Cardiovascular Diseases Fellowship Programs

Einstein Medical Center

5501 Old York Road, Levy 3232, Philadelphia, PA 19141

215-456-8991

figueredov@einstein.edu 
Abstract

Data on the distribution of dyssynchrony in subjects with normal ejection fraction and normal QRS are scarce. We studied 100 subjects with no known cardiac disease (52\% male, mean age $60 \pm 17$ years) using velocity vector imaging. Seventeen percent had septal to lateral (S-L) wall longitudinal delay $>75 \mathrm{~ms}, 63 \%$ of subjects had S-L wall radial delay $>75 \mathrm{~ms}$ and $25 \%$ had a circumferential opposing wall delay $>100 \mathrm{~ms}$. Those with circumferential opposing wall delay of $>100 \mathrm{~ms}$ had a lower ejection fraction $(57 \pm 5 \%$ vs. $62 \pm 5 \%, \mathrm{p}<0.05)$. In an additional group of 33 patients we compared the longitudinal dyssynchrony parameters as assessed by velocity vector imaging and tissue Doppler imaging and found them to be comparable. In conclusion, we find significant variation in time to peak velocities in subjects with no known cardiac disease, who had a normal left ventricular ejection fraction and QRS duration. Velocity vector imaging is comparable to tissue Doppler imaging. 
Introduction:

Cardiac resynchronization therapy (CRT) is the current standard of care for patients with left ventricular ejection fraction (LVEF) of $35 \%$ or less, New York Heart Association (NYHA) functional class III-IV, and QRS duration $>120 \mathrm{~ms}^{1}$. CRT has been shown to improve NYHA functional class, mortality, mitral regurgitation and left ventricular remodeling ${ }^{2,3}$. A wide QRS is used as a screening tool for mechanical dyssynchrony. However, not all patients with a wide QRS complex have mechanical dyssynchrony assessed by non-invasive echocardiographic (echo) techniques ${ }^{4}$ and further $30 \%$ of patients receiving CRT do not show significant improvement $^{2,5}$. Importantly, those with a narrow QRS complex can also have evidence of mechanical dyssynchrony ${ }^{4}$. In a recent multi-center trial 'Results of the Predictors of Response to CRT (PROSPECT) trial', color coded tissue Doppler imaging (TDI), among several other echo parameters was found not to be useful in real-world clinical settings for assessing mechanical dyssynchrony ${ }^{6}$.

Myocardial contraction and relaxation are complex processes involving longitudinal, circumferential, radial and torsional forces, among others. There is inherent electrical and mechanical inhomogeneity in the normally functioning heart ${ }^{7-10}$. Several studies have shown that the normal heart has regional variations in ejection fraction, wall thickening and timing of contraction of segments ${ }^{7-10}$. Although data on echo criteria for dyssynchrony in patients with left ventricular dysfunction are abundant, data on the variations in subjects with no known cardiac disease are scarce. Knowledge of the variations in timing of contraction of various segments will serve as a reference when studying the diseased heart. For example, variations in time to peak 
(TTP) velocities in subjects with no known cardiac disease could account for why TDI did not predict response to CRT in the PROSPECT trial ${ }^{6}$.

Unlike color-coded TDI which is dependant on the angle of the transducer and can only assess longitudinal velocities, a new B-mode echo technique called velocity vector imaging (VVI) is available $^{11}$. VVI is angle independent and can assess longitudinal, circumferential and radial velocities, and strain. The purpose of this study is two fold: 1) To assess whether variations are present in TTP velocities and strain among subjects without any known cardiac heart disease using VVI and 2) to directly compare color-coded TDI with VVI. 
Materials and Methods:

Three hundred and forty consecutive subjects who underwent 2D echocardiography at Albert Einstein Medical Center in Philadelphia between September 2007 and November 2007 on an Acuson Sequoia C512 (Siemens Medical Solutions, Inc.) were screened. Two hundred and forty subjects were excluded with the following exclusion criteria (1) history of coronary artery disease; (2) LVEF <55\%; (3) diastolic dysfunction greater than grade 1 (mitral early to late diastolic inflow peak velocity ratio $>0.8$, deceleration time of the mitral inflow $<200 \mathrm{msec}$, isovolumetric relaxation time $<60 \mathrm{msec}$, pulmonary venous systolic to diastolic peak velocity ratio $<1$, and mitral early inflow to early diastolic annular septal tissue peak velocity ratio $>9$, as listed in American Society of Echocardiography criteria ${ }^{12}$; (4) QRS duration >120 msec; (5) moderate or severe valvular heart disease (using Doppler echocardiographic parameters: central jet $>4 \mathrm{~cm} 2$ or jet area $>20 \%$ of left atrial area for mitral regurgitation, central jet width $>25 \%$ or vena contracta $>0.3 \mathrm{~cm} 2$ or pressure half-time $<500 \mathrm{msec}$ for aortic regurgitation, central jet area $>5 \mathrm{~cm} 2$ or proximal isovelocity surface radius $>0.5 \mathrm{~cm}$ for tricuspid regurgitation, jet size by color Doppler $>10 \mathrm{~mm}$ for pulmonary regurgitation for regurgitant lesions ${ }^{13}$; mean gradient $>20$ $\mathrm{mm} \mathrm{Hg}$ or aortic valve area $<1.5 \mathrm{~cm} 2$ or aortic jet velocity $>3 \mathrm{~m} / \mathrm{sec}$ for aortic stenosis, mitral valve area $<1.5 \mathrm{~cm} 2$ or mean gradient $>5 \mathrm{~mm} \mathrm{Hg}$ for mitral stenosis, tricuspid valve area $<1 \mathrm{~cm} 2$ or mean gradient $>5 \mathrm{~mm} \mathrm{Hg}$ or inflow time-velocity time integral $>60 \mathrm{~cm}$ or pressure half-time $>190 \mathrm{msec}$ for tricuspid stenosis, peak velocity $>3 \mathrm{~m} / \mathrm{sec}$ or peak gradient $>36 \mathrm{~mm} \mathrm{Hg}$ for pulmonic stenosis for stenotic lesions ${ }^{14}$; (6) pacemaker; (7) arrhythmias (8) hypertrophic cardiomyopathy; (9) pericardial effusion or disease; (10) poor-quality images in which the myocardium was not visible; and (11) admission to the intensive care unit. 
Additional echocardiograms of 35 consecutive patients (all-comers), who were not subjected to the above mentioned exclusion criteria, were studied to compare TDI and VVI measurements. The details of the VVI and TDI measurements are described below.

Patient demographics, clinical characteristics, laboratory data and QRS duration were collected (Table 1). Patient demographics collected were age, gender, and race. Clinical characteristics collected were any history of hypertension (blood pressure $>140 / 90 \mathrm{~mm} \mathrm{Hg}$ as defined by the seventh report of the Joint National Committee on Prevention, Detection, Evaluation, and Treatment of High Blood Pressure ${ }^{15}$ or use of antihypertensive agents); diabetes mellitus (fasting plasma glucose $>126 \mathrm{mg} / \mathrm{dL}$ per the American Diabetes Association ${ }^{16}$ or receipt of antidiabetic treatment); hypercholesterolemia (low-density lipoprotein level > $130 \mathrm{mg} / \mathrm{d}$ per the National Cholesterol Education Program ${ }^{17}$ or statin use) and smoking history. The longest QRS duration was recorded from the electrocardiogram. LVEF was calculated using the modified Simpson's rule ${ }^{18}$. Laboratory data obtained were hemoglobin and creatinine, which were measured closest to the time of echocardiography. This study was approved by the institutional research board of Einstein Medical Center, Philadelphia, PA.

The Acuson Sequoia C512 echo machine (Sequoia, Siemens Medical Solutions Inc., Mountain View, California), which has capabilities for both TDI and VVI, was used for the study. Images were captured using frame rates used for traditional 2D echocardiography (30-60 frames/sec). VVI uses a series of tracking algorithms whose details are described elsewhere ${ }^{11}$. In brief, the endocardial-myocardial interface is traced manually in a single frame on a digital cine loop. When the image is processed, a complex algorithm tracks each pixel, and the myocardial velocity vectors are displayed in cine format. The lengths of the vectors are proportional to the 
magnitude of velocity, and the direction of the arrows corresponds to the direction of myocardial motion. One cardiac cycle was analyzed if the RR intervals were regular, and an average of three beats was used if RR intervals were irregular (none of the study subjects had irregular RR intervals). Apical four-chamber, two-chamber, and short-axis views at the papillary muscle level were studied offline. In the apical four-chamber and two-chamber views, a trace was made (along the endocardial-myocardial interface) from the septal to lateral mitral annulus and from the inferior to anterior mitral annulus, respectively. In the short-axis view at the level of papillary muscles, a circumferential trace was made starting at the 12 o'clock position and ending at the same point in a clockwise direction, excluding the papillary muscles. Approximately one point per myocardial segment was used to draw the trace. A point of reference was placed at the apex in the two-chamber and four-chamber views to calculate longitudinal velocities and strain. The point of reference was moved to the LV cavity to calculate radial velocities. In the short-axis view, the point of reference was at the center of the left ventricle to calculate circumferential velocities and strain. Longitudinal velocity, longitudinal strain, and radial velocities were measured at the basal septal, basal lateral, basal anterior, and basal inferior walls in the apical four-chamber and two-chamber views. The circumferential velocities and strain were measured in the short-axis view at the papillary muscle level. Time to peak velocities and strain were calculated from the onset of the QRS complex to the peak systolic velocity or peak strain, respectively, during the ejection phase. We defined mechanical dyssynchrony as longitudinal opposing wall delay $>75$ msec by VVI on the basis of a prior study ${ }^{19}$. Because there are no published criteria for circumferential dyssynchrony and because we were looking at the maximum delay between all six segments in the short-axis view, not just the opposing wall delays, we used a higher number (maximum delay >100 msec) to define circumferential dyssynchrony. We used a value of $75 \mathrm{msec}$ for septal-to-lateral wall radial delay. Examples of 
longitudinal and circumferential LV dyssynchrony analysis using the above-mentioned VVI technique are illustrated in Figures 2 and 3, respectively.

For color coded TDI, the highest possible frame rate was used $(>100 \mathrm{mHz})$. The average of three beats was analyzed. Longitudinal velocities at the basal anterior, inferior, lateral and septal walls using TDI were measured. Care was taken to ensure that there was a distinct positive velocity in the systolic phase. If there was no positive velocity recorded, that trace was excluded. If there was more than one peak, the earliest peak was used to calculate TTP velocities. We defined mechanical dyssynchrony as an opposing wall delay $>75 \mathrm{~ms}$ by VVI (longitudinal and radial) based on a prior study ${ }^{11}$. Since there are no published criteria for circumferential dyssynchrony and since we were looking at the maximum delay between all 6 segments in the short axis view, and not just the opposing wall delays, we used a higher number (maximum delay $>100 \mathrm{~ms}$ ) to define circumferential dyssynchrony. Patients with poor color-coded TDI curves and patients with poor acoustic windows were excluded from the study. 
Statistical analysis:

Data were analyzed using the SPSS 10 (Chicago, IL, USA). Continuous data are presented as mean \pm SD. Means were compared using a two-tailed student t test. Chi-square test was used to compare categorical variables. A p value less than 0.05 were considered significant. Co-efficient of variation $(\mathrm{COV})$ was used to measure the inter and intra-observer variability on 20 random obese and non-obese subjects. 
$\underline{\text { Results: }}$

Description of subjects with normal EF and QRS $<120 \mathrm{~ms}$

The final cohort consisted of 100 patients with complete data. $52 \%$ were male, mean age $60 \pm 17$ years, mean QRS duration $86 \pm 12 \mathrm{~ms}$ and mean ejection fraction $(\mathrm{EF})$ was $61 \pm 6 \%$ (Table 1.)

\section{Longitudinal velocity:}

There were no significant differences in time to peak septal-lateral wall longitudinal velocities and time to peak inferior- anterior wall longitudinal velocities. The average septal to lateral wall and inferior-anterior wall delays were $34 \underline{+55} \mathrm{~ms}$ and $18 \pm 35 \mathrm{~ms}$, respectively.

\section{Radial velocity:}

The radial time to peak lateral wall velocity was significantly earlier than the time to peak radial septal velocity $(271 \pm 147 \mathrm{~ms}$ vs. $211 \pm 116 \mathrm{~ms}, \mathrm{p}<0.001)$. The absolute radial velocity of contraction of the anterior wall was significantly greater than the inferior wall $(2.2 \pm 1.4 \mathrm{~cm} / \mathrm{s}$ vs. $1.5 \pm 1.2 \mathrm{~cm} / \mathrm{s}, \mathrm{p}<0.05)$.

Circumferential velocity:

There were no significant differences in time to peak circumferential velocities among opposing walls in the short axis view. The average maximum delays between all the walls were $79 \pm 68 \mathrm{~ms}$. The average delay between anterior-inferior, anteroseptal-inferolateral and anterolateral inferoseptal walls were $43 \pm 58 \mathrm{~ms}, 49 \pm 53 \mathrm{~ms}$ and $50 \pm 58 \mathrm{~ms}$, respectively.

Variation in time to peak velocities:

Although there were no significant differences in overall time to peak velocities between opposing walls (except for radial septal- lateral wall time to peak velocities), there were 
significant variations in individual subjects. Of the 100 subjects only 22 had no longitudinal, radial or circumferential dyssynchrony. 3 subjects had longitudinal, radial and circumferential dyssynchrony. Those with all three forms of dyssynchrony had a significantly lower ejection fraction compared to those who did not have any dyssynchrony $(63 \pm 6 \%$ vs. $55 \%, \mathrm{p}<0.05)$. When the contribution of circumferential dyssynchrony was removed, the results were no longer significant. The remaining subjects had longitudinal, circumferential or radial dyssynchrony.

Variation in radial velocity:

There were significantly more variations in radial velocities when compared with longitudinal velocities (Fig 3). 63\% of subjects had S-L wall delay of radial velocities $>75 \mathrm{~ms}$. There were no significant differences in ejection fraction, age, QRS duration, longitudinal or circumferential velocities among those subjects with $\mathrm{S}-\mathrm{L}$ wall delay of $\leq 75 \mathrm{~ms}$ compared to those with S-L wall delay $>75 \mathrm{~ms}$.

\section{Variation in longitudinal velocity:}

Thirteen percent of subjects had S-L wall delay $>75 \mathrm{~ms}$ in longitudinal velocities by VVI (Fig. 4). These patients also had a significantly lower absolute velocity of contraction in the septum compared with the lateral wall $(2.7 \pm 1.2 \mathrm{~cm} / \mathrm{s}$ vs. $3.7 \pm 1.4 \mathrm{~cm} / \mathrm{s}, \mathrm{p}<0.05)$. There were no significant differences in ejection fraction, age, QRS duration, radial or circumferential velocities among those subjects with S-L wall delay of $\leq 75 \mathrm{~ms}$ compared to those with S-L wall delay $>75 \mathrm{~ms}$.

\section{Variation in circumferential velocity:}

Twenty five percent of subjects had $>100$ ms delay between time to peak circumferential velocities among all the walls in the short axis view. Those with $>100 \mathrm{~ms}$ delay had a significantly lower ejection fraction compared to those with $<100$ ms delay $(58 \pm 5 \%$ vs. $62 \pm 6 \%$, 
$\mathrm{p}<0.05$ ), although both ejections fractions were still in the normal range (Fig. 5, Table 2.). There were no significant differences in age, QRS duration, radial or longitudinal velocities among those subjects with a maximum delay of $<100 \mathrm{~ms}$ compared to those with a delay $>100 \mathrm{~ms}$.

\section{Variation in time to peak longitudinal and circumferential strain:}

The difference in time to peak longitudinal strain between the septal and lateral walls showed a similar pattern of variability when compared to longitudinal velocities. The mean difference in time to peak strain between the $S$ and L walls were $49 \pm 89 \mathrm{~ms}$ compared with $34 \pm 54 \mathrm{~ms}$ for time to peak $S-L$ velocities ( $p>0.05$ ). However, the maximum difference in time to peak circumferential strain between all the walls showed less variability than maximum difference in time to peak circumferential velocities. $(59 \pm 57 \mathrm{~ms}$ vs. $79 \pm 68 \mathrm{~ms}, \mathrm{p}<0.05)$.

Variation in time to peak velocities among those with no cardiac risk factors:

Only $23 \%(n=23)$ of patients in the cohort did not have any history of hypertension, diabetes, hypercholesterolemia and were non-smokers. Since these risk factors could potentially influence the distribution of dyssynchrony, we separately analyzed this group (mean age $47 \pm 19$ years, $26 \%$ male). Even in this healthy group, with normal EF, QRS $<120 \mathrm{~ms}$ and no cardiovascular risk factors, $13 \%$ had S-L wall longitudinal delay $>75 \mathrm{~ms}, 69 \%$ had radial S-L wall delay $>75 \mathrm{~ms}$ and $17 \%$ had circumferential maximum delay $>100 \mathrm{~ms}$. This mirrored the general distribution of dyssynchrony in the whole cohort of 100 patients.

\section{Comparison of VVI with TDI:}

We studied an additional 35 consecutive patients who underwent both VVI and TDI imaging. In two patients adequate TDI could not be obtained even after multiple attempts, due to inadequate frame rates or due to inconsistent TDI tracings, and were excluded. VVI could be performed and 
interpreted in all patients. This final cohort consisted of 33 patients with complete data. Sixtytwo percent were male, mean age $63 \pm 16$ years, mean QRS duration $76 \pm 40 \mathrm{~ms}$ and $22 \%$ had ejection fraction $(\mathrm{EF})<55 \%$. All patients (four) with S-L wall delay $>65 \mathrm{~ms}$ by TDI also had S-L wall delay by VVI (all greater than $75 \mathrm{~ms}$ ). Among the 27 patients with S-L wall delay $<65 \mathrm{~ms}$ by TDI (mean $24.7 \pm 22 \mathrm{~ms}$ ), 26 patients had S-L wall delays $<75 \mathrm{~ms}$ by VVI (mean $18.8 \pm 20 \mathrm{~ms}$ ). Although the other patient had a S-L wall delay $<65 \mathrm{~ms}$, there was an antero-posterior wall delay of $>65 \mathrm{~ms}$ by TDI.

Inter- and intra-observer variability was calculated for all VVI measurements in 20 random subjects. The coefficients of variation for longitudinal, radial, and circumferential time to peak velocities were $5 \%, 6 \%$, and $6 \%$, respectively, for interobserver variability and 5\%, 5\%, and $6 \%$, respectively, for intraobserver variability. The coefficients of variation for longitudinal and circumferential strain were $8 \%$ and $8 \%$, respectively, for interobserver variability and $7 \%$ and $8 \%$, respectively, for intraobserver variability. 


\section{Discussion:}

Although several single center studies have shown the utility of using echo criteria for assessing mechanical dyssynchrony ${ }^{20-23}$, it has not found wide clinical use. Part of the reason for this is that there are several different methods to assess mechanical dyssynchrony and several criteria have been proposed by different authors. Color coded TDI has been shown in several studies to predict left ventricular remodeling and response to $\mathrm{CRT}^{20-23}$. However, a recent multi-center trial (PROSPECT) failed to show the utility of CRT based on several echo criteria, including $\mathrm{TDI}^{6}$. This could be due to lack of strict standardization and uniformity in performing measurements, unlike in a single center study. Also, TDI may not be the best technique to predict response to CRT. While data on timing of regional contraction in those with LV dysfunction is abundant, similar data on subjects with normal EF is scarce. There is normal apex to base spread of electrical activity and a similar apex to base spread of myocardial contraction ${ }^{24}$. There can be significant regional heterogeneity in electrical and mechanical activation of the normal heart ${ }^{9,24,}$ 25. The degree of heterogeneity can vary and some normal healthy subjects could meet criteria for LV dyssynchrony. Therefore, it is important to know what the baseline variations in regional timing of contraction of the normal heart are in order to be able to establish criteria in the diseased state. Although initial studies by Yu et. al. ${ }^{26}$ found low variability in TDI measurements in healthy patients, other studies found more variations in normal subjects ${ }^{27}$. Zwanenburg et al, studied MRI derived time to peak strain and time to onset of contraction in healthy volunteers and found that there is heterogeneity in contraction between different segments of the heart, with the range being between $200-400 \mathrm{~ms}$ for onset of time to peak contraction $^{10}$. A recent study by Miyazaki et. al. found significant overlap of TDI measurements in four groups of subjects: those with normal LV function and a narrow QRS, subjects with 
normal LV function and left bundle branch block, subjects with LV dysfunction and narrow QRS, and subjects with wide QRS and depressed LV function ${ }^{28}$.

In our study of 100 subjects with normal LVEF and narrow QRS, the average S-L wall delay for

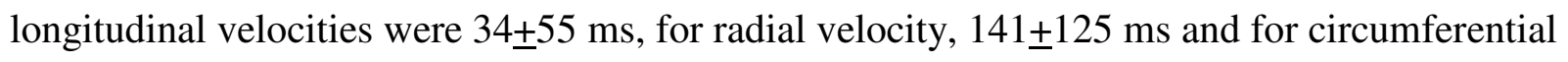
velocities, the average opposing wall delays were $43 \pm 58 \mathrm{~ms}$ between the anterior and inferior wall, $49 \pm 53 \mathrm{~ms}$ between the anteroseptal and inferolateral wall and $50 \pm 58 \mathrm{~ms}$ between the anterolateral and inferoseptal walls. Using the prior published criteria for dyssynchrony by VVI ${ }^{19}$ (S-L wall delay $>75 \mathrm{~ms}$ for longitudinal velocities), $13 \%$ of subjects met criteria for longitudinal dyssynchrony. There were no significant differences between the groups with and without dyssynchrony suggesting that this may be the normal spread of opposing wall delay values. The spread of opposing wall time to peak radial velocities were even wider. Sixty three percent of subjects had S-L wall radial delay $>75 \mathrm{~ms}$. Criteria for dyssynchrony for time to peak radial velocities have not been established. This wide spread would suggest that the cut off value for dyssynchrony for radial velocities would be higher than for longitudinal velocities. The spread of values for circumferential time to peak velocities were in-between those for radial and longitudinal velocities. Twenty-five percent of subjects had maximum opposing wall delay $>100$ ms. In contrast to the subjects above who despite significant variation in velocities demonstrated no differences between groups, subjects with a maximum opposing delay $>100 \mathrm{~ms}$. These subjects also had a significantly lower left ventricular ejection fraction (58 $\underline{5} \%$ vs. $62 \pm 5 \%$; $\mathrm{p}=0.001)$ compared to those with maximum opposing wall delay $<100 \mathrm{~ms}$. Although both these ejection fractions were still within the normal range, the results were statistically significant. It is well known that one of the major contributors to robust left ventricular emptying is the twisting or wringing motion of the heart ${ }^{25}$. It is conceivable that those with greater degrees of inhomogeneity in circumferential twist would have lower left ventricular ejection fractions. 
Whether these patients have a greater chance of progression to significant left ventricular dysfunction is not known and requires further study.

We also compared those patients who did not have any evidence of radial, longitudinal or circumferential dyssynchrony (22 subjects) with those who had evidence of all three forms of dyssynchrony (3 subjects). The only statistically significant difference between these groups was

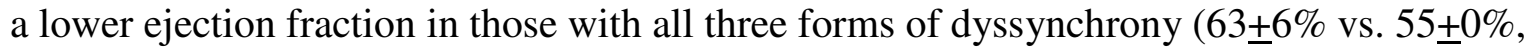
$\mathrm{p}<0.05)$. Once the contribution of circumferential dyssynchrony was removed, the results were no longer statistically significant. Again, it is unclear whether these subjects will go on to have significant left ventricular dysfunction in the future. Although the difference in time to peak longitudinal strain between opposing walls showed a similar spread to TTP longitudinal velocities, the difference in time to peak circumferential strain showed significantly less variation. Thus, as suggested by Miyazaki et $\mathrm{al}^{28}$, it is possible that time to peak strain measurements may by more accurate to assess dyssynchrony. However, additional trials are needed to answer this question.

Similar to the wide spread of opposing wall delays in subjects without heart disease, those with left ventricular dysfunction may also have a normal spread of opposing wall delays, and thus all of them may not respond to CRT. If a subject with normal left ventricular function and evidence of dyssynchrony at baseline develops left ventricular dysfunction and the degree of dyssynchrony persists, it is unclear whether this patient will respond to CRT. On the other hand, if someone with preserved left ventricular function and no dyssynchrony at baseline develops left ventricular systolic dysfunction and dyssynchrony subsequently develops, they may derive benefit from CRT. Since cardiac mechanics are complex, defining dyssynchrony by opposing wall delays in one dimension may be too simplistic. Some degree of dyssynchrony may be of no hemodynamic consequence to one individual, but may cause significant hemodynamic 
disturbance in other subjects. Thus, using a blanket cut off value to define response to dyssynchrony may not be ideal. Costa et al. also showed that a significant fraction of their study group with preserved ejection fraction and normal QRS width had dyssynchrony by M-Mode and tissue Doppler echocardiography ${ }^{29}$. With the increasing use of 3-dimensional echocardiography in the assessment of left ventricular dyssynchrony and cardiac resynchronization therapy, it would be interesting to see if similar results are obtained when using this modality ${ }^{30,31}$. Sengupta et. al have studied blood flow hemodynamics and have shown characteristic filling and emptying patters of left ventricular blood flow using ultrasonic digital particle imaging velocimetry ${ }^{32}$. Thus, looking at the end result of left ventricular mechanics, namely efficient patterns of blood flow within the left ventricle may be a more ideal way to assess left ventricular synchrony than looking at the mechanical delay between LV walls.

\section{Comparison of VVI with TDI:}

VVI is angle independent, and can be performed without the need for very high frame rates. It also allows assessment of radial and circumferential dyssynchrony in addition to longitudinal dyssynchrony. Data on the use of VVI for dyssynchrony are scarce. Cannesson and colleagues have shown that an opposing wall delay $>75 \mathrm{~ms}$ by VVI predicts LV remodeling after CRT ${ }^{19}$. They however did not make a direct comparison between TDI and VVI. Based on that study, we used a cut off of $75 \mathrm{~ms}$ between septal and lateral wall TTP longitudinal velocities to define dyssynchrony. Among those patients whose TDI could be analyzed, everyone with septal to lateral wall delay $>65 \mathrm{~ms}$ also had S-L wall delay $>75 \mathrm{~ms}$ by VVI. Similarly, all but one patient with S-L wall delay $<65 \mathrm{~ms}$ by TDI had no longitudinal dyssynchrony by VVI. This patient had anterior to posterior wall delay by TDI (>65 ms) and thus technically has mechanical 
dyssynchrony. Two patients had poor quality TDI tracings that could not be analyzed. VVI could be performed and interpreted in all patients studied. Thus VVI is an attractive alternative to TDI for assessing mechanical dyssynchrony, and does not carry some limitations of TDI. 


\section{Limitations:}

Although consecutive patients undergoing echocardiography with normal EF and QRS duration were studied, this cohort does not represent a truly healthy population with a significant number of patients having cardiovascular risk factors. It is possible that these risk factors could have influenced the distribution of dyssynchrony. However, we separately analyzed 23 subjects without any cardiovascular risk factors and the distribution of dyssynchrony in this healthy group was similar to the overall cohort of patients. Even in the 23 healthy subjects, given the older age of the population, it is possible that unaccounted confounders, such as occult coronary artery disease could influence dyssynchrony. The effect of body mass index on the distribution of dyssynchrony was not assessed in this study. We have shown in one of our previous studies that there was an increased prevalence of dyssynchrony among the obese with no known history of significant cardiac disease ${ }^{33}$. Since a lower frame rate was used for VVI, it is conceivable that some very rapid velocities seen with TDI may not be recorded. However, comparison between the time to peak velocities between two walls should still be valid. MRI studies looking at the heterogeneity in LV contraction used frame rates ranging from $14-35 \mathrm{~ms}^{7,10}$. This is comparable with the 30-60 f/s (16-33 ms) used in this study. However, it is lower than frame rates used for TDI ( $>100 \mathrm{f} / \mathrm{s}$ or $<10 \mathrm{~ms})$. 


\section{Conclusions:}

In summary, there appears to be normal heterogeneity in opposing wall delays in adult subjects without apparent heart disease. The variation in opposing wall delay seems to be highest for radial velocities and least for longitudinal velocities. Even in the normal range of left ventricular ejection fractions, those with circumferential dyssynchrony seems to have lower ejection fractions, highlighting the contribution of left ventricular twist towards the overall left ventricular function. Knowing the normal spread of velocities is important in order to be able to set appropriate limits in the diseased state. Since there is a normal heterogeneity in opposing wall delays, and since a recent large trial has shown that echo-guided resynchronization was not able to predict response to CRT, it may be worthwhile to explore alternative indicators of dyssynchrony such as left ventricular blood flow dynamics. Alternatively, instead of focusing on longitudinal velocities, it may be useful to integrate multiple measures of dyssynchrony as radial and circumferential time to peak velocities. Another area that will be interesting to study is whether healthy subjects with mechanical dyssynchrony will eventually develop left ventricular dysfunction. When comparing VVI with TDI, VVI appears as good as TDI for assessing longitudinal dyssynchrony, and may be useful in evaluating patients with poor quality TDI images. In addition, VVI can be used to detect circumferential and radial dyssynchrony. VVI is an attractive alternative to TDI for assessing mechanical dyssynchrony and does not carry some of the technical limitations of TDI. 
References

1. Epstein AE, DiMarco JP, Ellenbogen KA, Estes NA, 3rd, Freedman RA, Gettes LS, et al. ACC/AHA/HRS 2008 Guidelines for Device-Based Therapy of Cardiac Rhythm Abnormalities: a report of the American College of Cardiology/American Heart Association Task Force on Practice Guidelines (Writing Committee to Revise the ACC/AHA/NASPE 2002 Guideline Update for Implantation of Cardiac Pacemakers and Antiarrhythmia Devices) developed in collaboration with the American Association for Thoracic Surgery and Society of Thoracic Surgeons. J Am Coll Cardiol 2008;51(21):e162.

2. Bristow MR, Saxon LA, Boehmer J, Krueger S, Kass DA, De Marco T, et al. Cardiacresynchronization therapy with or without an implantable defibrillator in advanced chronic heart failure. N Engl J Med 2004;350(21):2140-50.

3. Cleland JG, Daubert JC, Erdmann E, Freemantle N, Gras D, Kappenberger L, et al. The effect of cardiac resynchronization on morbidity and mortality in heart failure. $\mathrm{N}$ Engl $\mathbf{J}$ Med 2005;352(15):1539-49.

4. Bleeker GB, Schalij MJ, Molhoek SG, Verwey HF, Holman ER, Boersma E, et al. Relationship between QRS duration and left ventricular dyssynchrony in patients with end-stage heart failure. J Cardiovasc Electrophysiol 2004;15(5):544-9. 
5. Abraham WT, Fisher WG, Smith AL, Delurgio DB, Leon AR, Loh E, et al. Cardiac resynchronization in chronic heart failure. N Engl J Med 2002;346(24):1845-53.

6. Chung ES, Leon AR, Tavazzi L, Sun JP, Nihoyannopoulos P, Merlino J, et al. Results of the Predictors of Response to CRT (PROSPECT) trial. Circulation 2008;117(20):260816.

7. Fonseca CG, Oxenham HC, Cowan BR, Occleshaw CJ, Young AA. Aging alters patterns of regional nonuniformity in LV strain relaxation: a 3-D MR tissue tagging study. Am J Physiol Heart Circ Physiol 2003;285(2):H621-30.

8. Young AA, Imai H, Chang $\mathrm{CN}$, Axel L. Two-dimensional left ventricular deformation during systole using magnetic resonance imaging with spatial modulation of magnetization. Circulation 1994;89(2):740-52.

9. Bogaert J, Rademakers FE. Regional nonuniformity of normal adult human left ventricle. Am J Physiol Heart Circ Physiol 2001;280(2):H610-20.

10. Zwanenburg JJ, Gotte MJ, Kuijer JP, Heethaar RM, van Rossum AC, Marcus JT. Timing of cardiac contraction in humans mapped by high-temporal-resolution MRI tagging: early onset and late peak of shortening in lateral wall. Am J Physiol Heart Circ Physiol 2004;286(5):H1872-80.

11. Jackson J,Houle H. SyngoVelocityVector Imaging.Available at: http://www. 
medical.siemens.com/siemens/en_US/gg_us_FBAs/files/misc_downloads/ Whitepaper_Jackson.pdf. Accessed February 14, 2012.

12. Nagueh SF, Appleton CP, Gillebert CT, Marino PN, Oh JK, Smiseth OA, et al. Recommendations for the evaluation of left ventricular diastolic function by echocardiography. J Am Soc Echocardiogr 2009;22:107-33

13. Zoghbi WA, Enriquez-Sarano M, Foster E, Grayburn PA, Kraft CD, Levine RA, et al. Recommendations for evaluation of the severity of native valvular regurgitation with two-dimensional and Doppler echocardiography. J Am Soc Echocardiogr 2003;16:777-802.

14. Baumgartner H, Hung J, Bermejo J, Chambers JB, Evangelista A, Griffin BP, et al. Echocardiographic assessment of valve stenosis: EAE/ASE recommendations for clinical practice. J Am Soc Echocardiogr 2009;22:1-23

15. Chobanian AV, Bakris GL, Black HR, CushmanWC, Green LA, Izzo JL Jr., et al. The JNC 7 report. JAMA 2003;289:2560-72

16. American Diabetes Association. Position statement. Standards of medical care in diabetes-2010. Diabetes Care 2010;33(suppl):S11-61

17. Expert Panel on Detection, Evaluation, and Treatment of High Blood Cholesterol in Adults. Third report of the Expert Panel on Detection, Evaluation, and Treatment of High 
Blood Cholesterol in Adults (Adult Treatment Panel III): final report. Available at: http://www.nhlbi.nih.gov/guidelines/cholesterol/atp3full.pdf.

18. Lang RM, Bierig M, Devereux RB, Flachskampf FA, Foster E, Pellikka PA, et al. Chamber Quantification Writing Group; American Society of Echocardiography's Guidelines and Standards Committee; European Association of Echocardiography. Recommendations for chamber quantification: a report from the American Society of Echocardiography's Guidelines and Standards Committee and the Chamber Quantification Writing Group, developed in conjunction with the European Association of Echocardiography, a branch of the European Society of Cardiology. J Am Soc Echocardiogr 2005;18:1440-63.

19. Cannesson M, Tanabe M, Suffoletto MS, Schwartzman D, Gorcsan J, 3rd. Velocity vector imaging to quantify ventricular dyssynchrony and predict response to cardiac resynchronization therapy. Am J Cardiol 2006;98(7):949-53.

20. Yu CM, Chau E, Sanderson JE, Fan K, Tang MO, Fung WH, et al. Tissue Doppler echocardiographic evidence of reverse remodeling and improved synchronicity by simultaneously delaying regional contraction after biventricular pacing therapy in heart failure. Circulation 2002;105(4):438-45.

21. Bax JJ, Bleeker GB, Marwick TH, Molhoek SG, Boersma E, Steendijk P, et al. Left ventricular dyssynchrony predicts response and prognosis after cardiac resynchronization therapy. J Am Coll Cardiol 2004;44(9):1834-40. 
22. Bax JJ, Marwick TH, Molhoek SG, Bleeker GB, van Erven L, Boersma E, et al. Left ventricular dyssynchrony predicts benefit of cardiac resynchronization therapy in patients with end-stage heart failure before pacemaker implantation. Am J Cardiol 2003;92(10):1238-40.

23. Yu CM, Fung JW, Zhang Q, Chan CK, Chan YS, Lin H, et al. Tissue Doppler imaging is superior to strain rate imaging and postsystolic shortening on the prediction of reverse remodeling in both ischemic and nonischemic heart failure after cardiac resynchronization therapy. Circulation 2004;110(1):66-73.

24. Sengupta PP, Khandheria BK, Korinek J, Wang J, Jahangir A, Seward JB, et al. Apex-tobase dispersion in regional timing of left ventricular shortening and lengthening. $\mathbf{J}$ Am Coll Cardiol 2006;47(1):163-72.

25. Sengupta PP, Korinek J, Belohlavek M, Narula J, Vannan MA, Jahangir A, et al. Left ventricular structure and function: basic science for cardiac imaging. J Am Coll Cardiol 2006;48(10):1988-2001.

26. Yu CM, Lin H, Zhang Q, Sanderson JE. High prevalence of left ventricular systolic and diastolic asynchrony in patients with congestive heart failure and normal QRS duration. Heart 2003;89(1):54-60. 
27. Poerner TC, Goebel B, Geiger T, Haghi D, Borggrefe M, Haase KK. Physiological range of mechanical synchronicity of the human heart: comparison between different echocardiographic assessment modalities. Ultrasound Med Biol 2005;31(9):1163-72.

28. Miyazaki C, Powell BD, Bruce CJ, Espinosa RE, Redfield MM, Miller FA, et al. Comparison of echocardiographic dyssynchrony assessment by tissue velocity and strain imaging in subjects with or without systolic dysfunction and with or without left bundlebranch block. Circulation 2008;117(20):2617-25.

29. Costa S, O'Mara J, Young C, Gama M, Palac R. The echocardiographic assessment of dyssynchrony: insights from a consecutive series of general cardiology patients with normal LVEF and narrow QRS. Echocardiography 2009 May;26(5):534-40

30. Wang H, Shuraih M, Ahmad M. Real time three-dimensional echocardiography in assessment of left ventricular dyssynchrony and cardiac resynchronization therapy. Echocardiography 2012 Feb;29(2):192-9.

31. Tani T, Sumida T, Tanabe K, Ehara N, Yamaguchi K, Kawai J, et al. Left ventricular systolic dyssynchrony index by three-dimensional echocardiography in patients with decreased left ventricular function: comparison with tissue Doppler echocardiography. Echocardiography 2012 Mar;29(3):346-52.

32. Sengupta PP, Khandheria BK, Korinek J, Jahangir A, Yoshifuku S, Milosevic I, et al. Left ventricular isovolumic flow sequence during sinus and paced rhythms: new insights 
from use of high-resolution Doppler and ultrasonic digital particle imaging velocimetry. J Am Coll Cardiol 2007;49(8):899-908.

33. Purushottam B, Parameswaran AC, Figueredo VM. Dyssynchrony in obese subjects without a history of cardiac disease using velocity vector imaging. J Am Soc Echocardiogr. 2011 Jan;24(1):98-106. 
Legends:

Figure 1. Velocity vector imaging in the apical four-chamber view.

Figure 2. Velocity vector imaging in the short axis view.

Figure 3. Variation in time to peak velocities.

Figure 4. Distribution of longitudinal time to peak septal to lateral wall delays.

Figure 5. Left ventricular ejection fraction in those with and without circumferential dyssynchrony. 
Table 1. Baseline characteristics of 100 people with normal ejection fraction and normal QRS duration.

\begin{tabular}{|c|c|}
\hline Males & $52 \%$ \\
\hline Age & $60 \pm 17$ years \\
\hline QRS Duration & $86.2 \pm 12.6 \mathrm{~ms}$ \\
\hline Ejection Fraction & $61 \pm 6 \%$ \\
\hline Hypertension & $63 \%$ \\
\hline Diabetes & $30 \%$ \\
\hline Hypercholesterolemia & $26 \%$ \\
\hline Smoker & $11 \%$ \\
\hline Hemoglobin (g/dL) & $12.5 \pm 2.1$ \\
\hline Creatinine (mg/dL) & $1.6 \pm 1.5$ \\
\hline Radial maximum TTP delay & $241.5 \pm 139 \mathrm{~ms}$ \\
\hline Radial TTP S-L delay & $141.8 \pm 125 \mathrm{~ms}$ \\
\hline Radial TTP A-I delay & $120.4 \pm 100 \mathrm{~ms}$ \\
\hline Circumferential TTP maximum delay & $79 \pm 68 \mathrm{~ms}$ \\
\hline $\begin{array}{l}\text { Circumferential TTP delay between A and I } \\
\text { walls }\end{array}$ & $43.3 \pm 58 \mathrm{~ms}$ \\
\hline $\begin{array}{l}\text { Circumferential TTP delay between IS and AL } \\
\text { walls }\end{array}$ & $49.7 \pm 53 \mathrm{~ms}$ \\
\hline Circumferential TTP delay between AL and IS & $50.1 \pm 58 \mathrm{~ms}$ \\
\hline Longitudinal maximum delay & $95.9 \pm 70 \mathrm{~ms}$ \\
\hline Longitudinal TTP delay between S and L walls & $34.6 \pm 55 \mathrm{~ms}$ \\
\hline Longitudinal TTP delay between A and I walls & $18.2 \pm 35 \mathrm{~ms}$ \\
\hline
\end{tabular}

TTP- Time to peak

S, L, A, I, AS, AL, IL, IS- Septal, Lateral, Anterior, Inferior, Anteroseptal, Anterolateral, Inferolateral and Inferoseptal walls respectively. 
Table 2. Relationship of left ventricular ejection fraction (EF) with time to peak (TTP) opposing wall delays.

Left ventricular EF in those with circumferential maximum TTP delay (CVVI) $>100 \mathrm{~ms}$ vs. $\leq 100 \mathrm{~ms}$

\begin{tabular}{|l|c|c|c|}
\hline & $\mathrm{N}$ & Mean $\mathrm{EF} \pm \mathrm{SD}$ & $\mathrm{P}$ value \\
\cline { 1 - 3 } $\mathrm{CVVI}>100$ & 27 & $58.2 \pm 5 \%$ & \multirow{2}{*}{0.001} \\
\cline { 1 - 2 } $\mathrm{CVVI} \leq 100$ & 73 & $62.5 \pm 6 \%$ & \\
\hline
\end{tabular}

Left ventricular EF in those with longitudinal TTP septal -lateral wall delay (LVVI) >75 ms vs. $\leq 75 \mathrm{~ms}$

\begin{tabular}{|c|c|c|c|}
\hline & $\mathrm{N}$ & Mean $\mathrm{EF} \pm \mathrm{SD}$ & $\mathrm{P}$ value \\
\cline { 1 - 3 } $\mathrm{LVVI} \leq 75$ & 87 & $61.4 \pm 5$ & 0.7 \\
\cline { 1 - 3 } $\mathrm{LVVI}>75$ & 13 & $60.7 \pm 7$ & \\
\hline
\end{tabular}

Left ventricular EF in those with radial TTP septal -lateral wall delay (RVVI) >75 ms vs. $<75 \mathrm{~ms}$

\begin{tabular}{|l|c|c|c|}
\hline \hline & $\mathrm{N}$ & Mean \pm SD & P value \\
\cline { 1 - 3 } $\mathrm{RVVI} \leq 75$ & 36 & $61.5 \pm 6$ & \multirow{2}{*}{0.8} \\
\cline { 1 - 3 } $\mathrm{RVVI}>75$ & 64 & $61.2 \pm 5$ & \\
\hline
\end{tabular}


Figure1

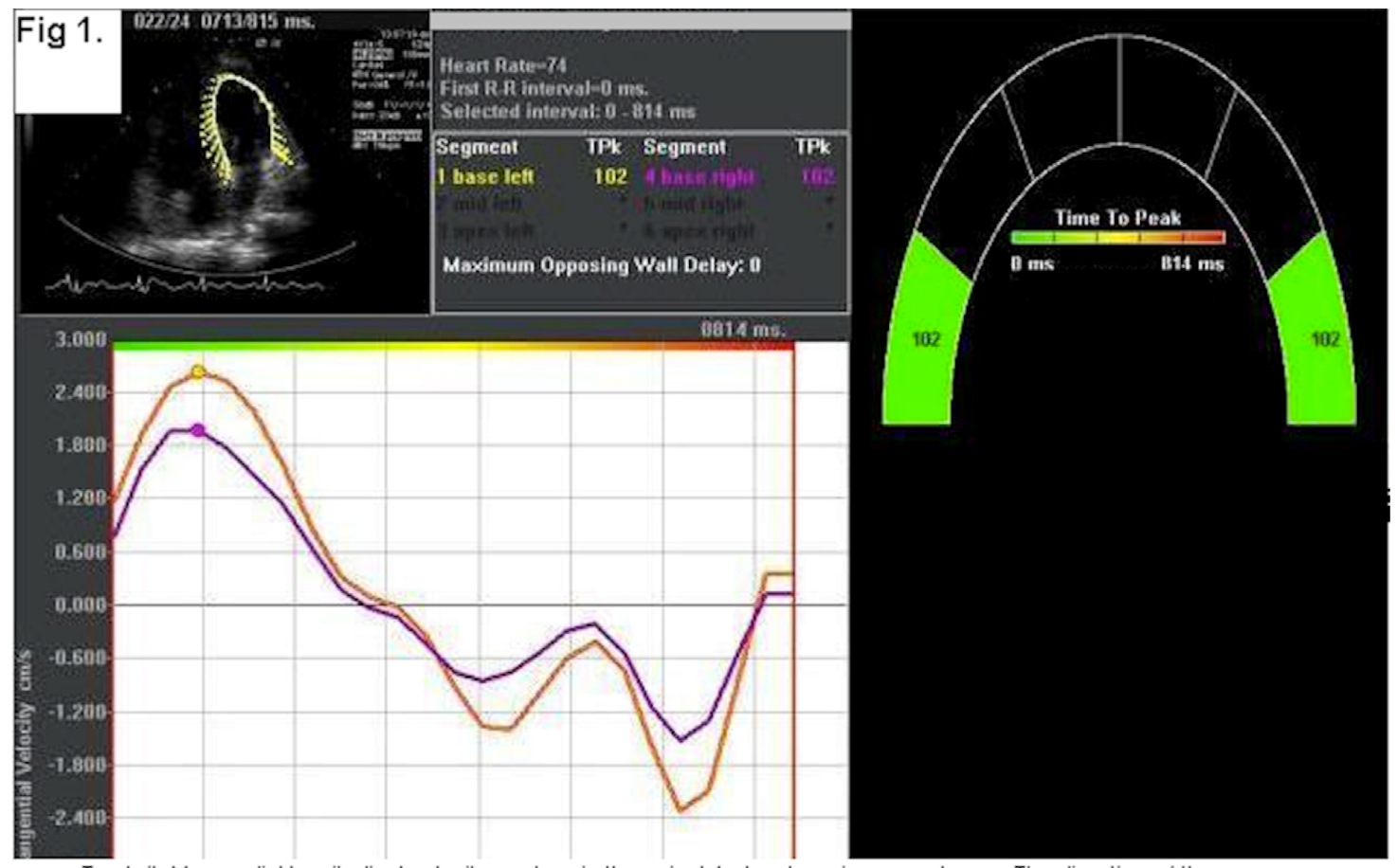

Top left: Myocardial longitudinal velocity vectors in the apical 4-chamber view are shown. The direction of the arrows correspond to the direction of contraction and the length of arrows is proportional to the velocity of contraction. Bottom left: Velocity curves for the basal septal and basal lateral walls are shown. Top right: The time to peak delay between these walls is shown in graphic format. Note that there is no delay between the basal septal and lateral walls. 
Figure 2
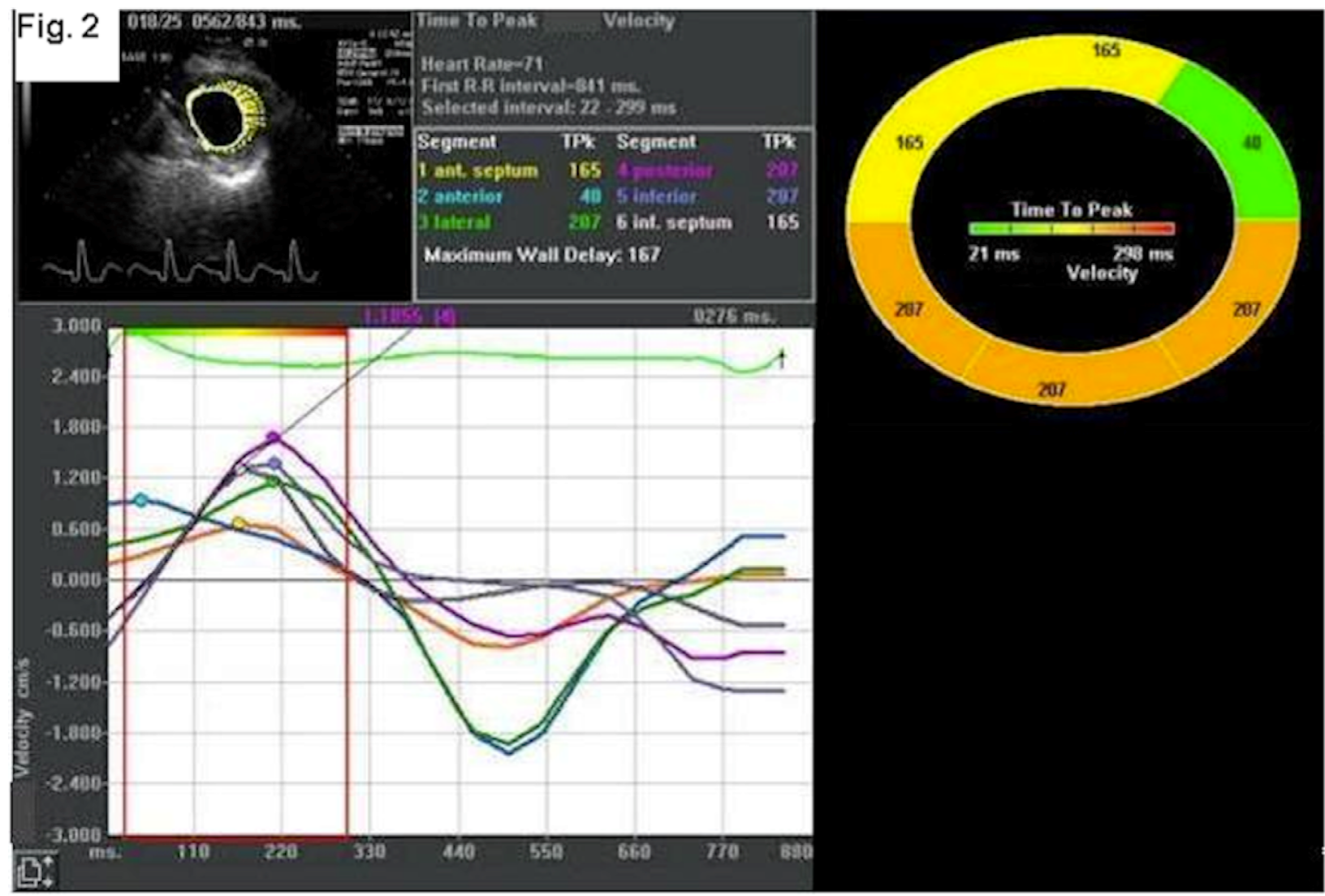

Top left: Circumferential velocity vectors in the short axis view are shown. The direction of the arrows correspond to the direction of contraction and the length of arrows is proportional to the velocity of contraction. Bottom left: The velocity curves for various myocardial segments in the short axis view are shown. Top right: The time to attain peak velocity of each myocardial segment is shown in graphic format. Note that the maximum delay between all the walls is $>100 \mathrm{~ms}$. 
Figure 3

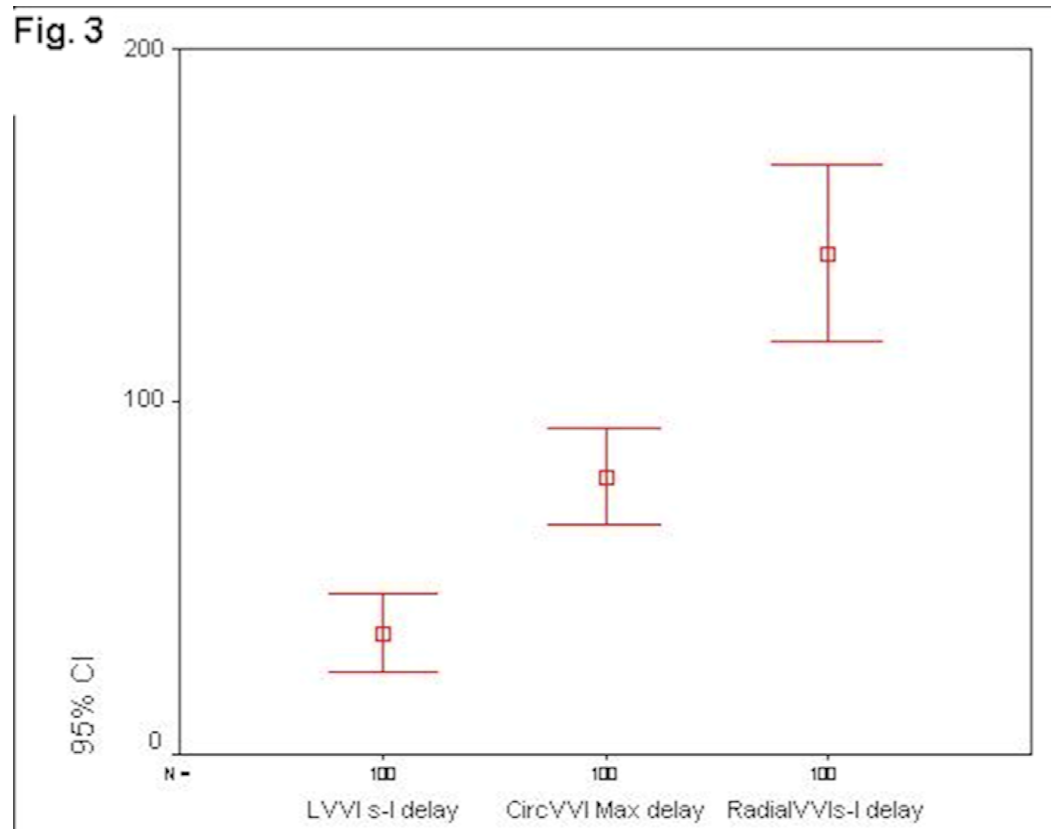

Distribution (mean $\pm 95 \%$ confidence interval) oflongtudinal (LVVI s-1 delay) and radial (RadialVVIs-1 delay) time to peak septal -lateral wall delays. The circumferential (CircVVI Max delay) time to peak delay is the maximum delay between all opposing walls in the short axis view. The radial and circumferential time to peak velocities show more variation than longitudinal time to peak velocities. 
Figure 4

Fig. 4

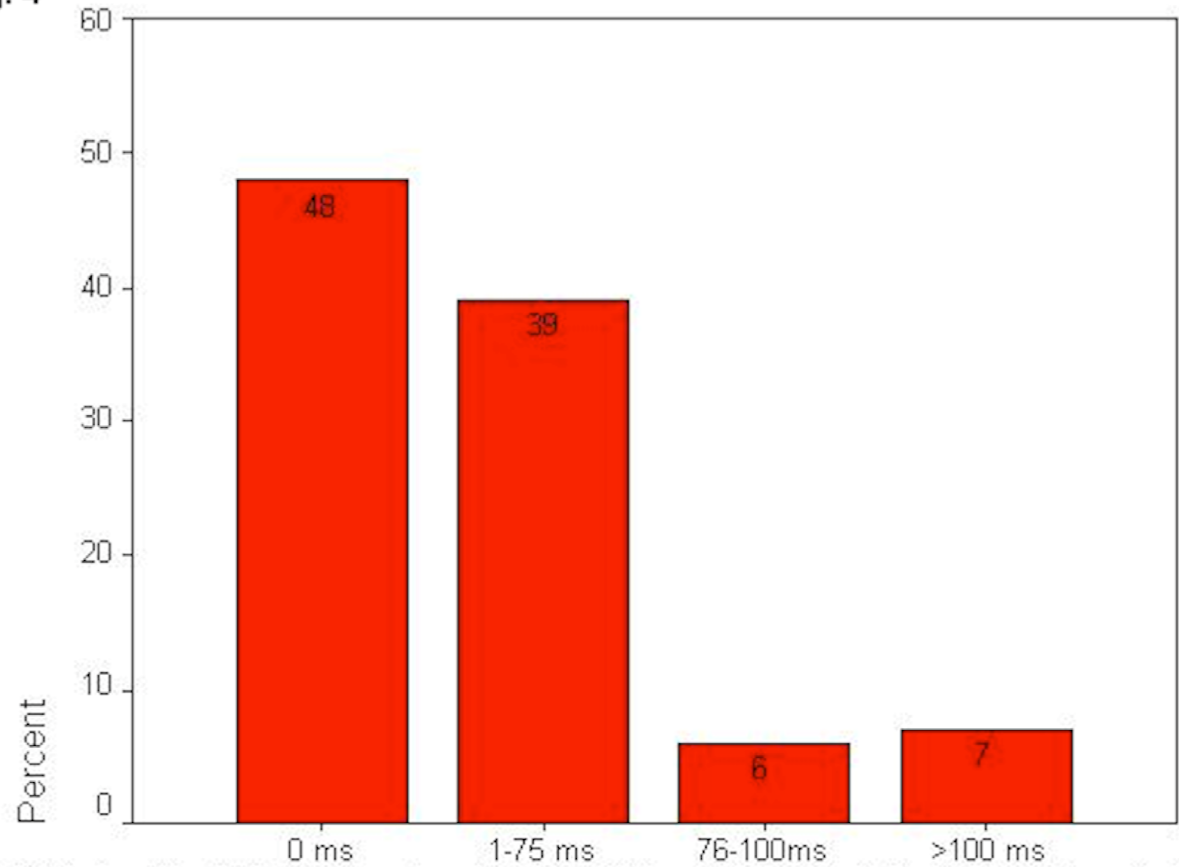

Distribution of longitudinal time to peak septal - lateral (S-L) wall delays. Note that in $13 \%$ of healthy subjects, the S-L wall delay is $>75 \mathrm{~ms}$. In $48 \%$, the $S-L$ wall delay is zero. 
Figure 5

Fig. 5

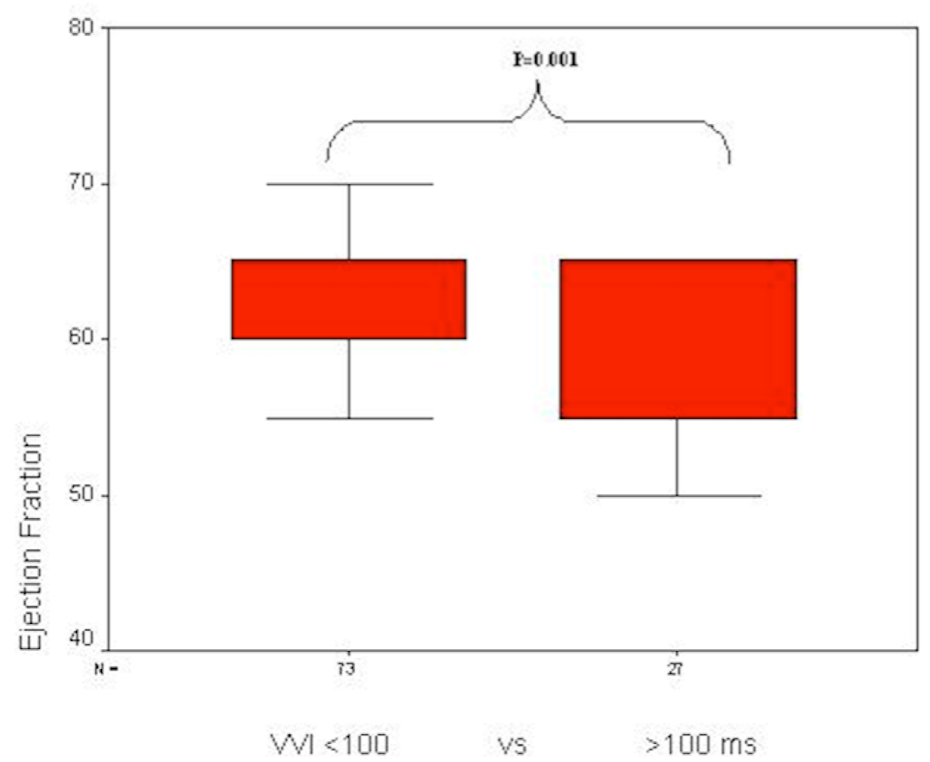

Left ventricular ejection fraction in those with circumferential maximum delay $(V \mathrm{~V} \mid) \leq 100 \mathrm{~ms}$ vs $\$ 100 \mathrm{~ms}$. Note that in those with $\mathrm{VV} /<100 \mathrm{~ms}$, the ejection fraction is higher $(p=0.001)$. 